\title{
Perfect Matching Preservers
}

\author{
Richard A. Brualdi \\ Department of Mathematics \\ University of Wisconsin - Madison \\ Madison, WI 53706, USA \\ brualdi@math.wisc.edu \\ Martin Loebl* \\ Department of Applied Mathematics \\ Institute for Theoretical Computer Science \\ Charles University \\ Malostranské náměstí 25, 11800 \\ Prague, Czech Republic \\ loebl@kam.mff.cuni.cz \\ Ondřej Pangrác* \\ Department of Applied Mathematics \\ Institute for Theoretical Computer Science \\ Charles University \\ Malostranské náměstí 25, 11800 \\ Prague, Czech Republic \\ pangrac@kam.mff.cuni.cz
}

Submitted: Nov 15, 2004; Accepted: Oct 19, 2006; Published: Oct 31, 2006

AMS Subject Classification:05C20, 05C50, 05C70

\begin{abstract}
For two bipartite graphs $G$ and $G^{\prime}$, a bijection $\psi: E(G) \rightarrow E\left(G^{\prime}\right)$ is called a (perfect) matching preserver provided that $M$ is a perfect matching in $G$ if and only if $\psi(M)$ is a perfect matching in $G^{\prime}$. We characterize bipartite graphs $G$ and $G^{\prime}$ which are related by a matching preserver and the matching preservers between them.
\end{abstract}

${ }^{*}$ Supported by Ministry of Education of Czech Republic as project LN00A056. 


\section{Introduction}

A subset $M \subseteq E(G)$ of the edge set $E(G)$ of a graph $G$ is called a matching provided that no two edges in $M$ have a vertex in common. A perfect matching $M$ is a matching with the property that each vertex of $G$ is incident with an edge in $M$. For $k$ a positive integer, a graph $G$ is $k$-extendable provided that $G$ has a matching of size $k$ and every matching in $G$ of size at most $k$ can be extended to a perfect matching in $G$.

In this paper we characterize the bipartite graphs $G$ and $G^{\prime}$ that are related by a matching preserver and so, with appropriate labeling of edges, have the same perfect matchings. We will achieve this by a full description of matching preservers defined as follows: A bijection $\psi: E(G) \rightarrow E\left(G^{\prime}\right)$ is matching preserving, or is a matching preserver, provided that $M$ is a perfect matching in $G$ if and only if $\psi(M)$ is a perfect matching in $G^{\prime}$. Matching preservers for bipartite graphs $G$ were investigated in [2] (see also [1]) in the context of the diagonals of a matrix and the associated diagonal hypergraph. Let $A$ be the bi-adjacency matrix of $G$. Then $A$ is a $(0,1)$-matrix, and the matchings of $G$ are in one-to-one correspondence with the permutation matrices $P$ satisfying $P \leq A$ (entrywise order). The property that $G$ is 1-extendable is equivalent to the property that the biadjacency matrix $A$ has total support. The property that $G$ is connected and 1-extendable is equivalent to the property that $A$ is fully indecomposable. See [3] for a discussion of these matrix properties. The vertices of the hypergraph mentioned above correspond to the edges of $G$ (the positions of the 1's in $A$ ) and the hyperedges are the perfect matchings of $G$ (the permutations matrices $P \leq A$, more properly, the set of the $n$ positions of $P$ that are occupied by 1 's).

Let $G$ be a connected, 1-extendable, bipartite graph with parts $X$ and $Y$ of size $n$. The edges of $G$ are pairs $x y$ of vertices with $x \in X$ and $y \in Y$. Let $u$ and $v$ be vertices belonging to different parts of $G$ such that $\{u, v\}$ forms a vertex cut of $G$. Thus there are bipartite graphs $G_{1}$ with parts $X_{1} \subseteq X, Y_{1} \subseteq Y$, and $G_{2}$ with parts $X_{2} \subseteq X, Y_{2} \subseteq Y$, such that $X_{1} \cap X_{2}=\{u\}$ and $Y_{1} \cap Y_{2}=\{v\}$ and each edge of $G$ belongs to either $G_{1}$ or $G_{2}$ (if $u v$ is an edge of $G$, then $u v$ is the only common edge of $G_{1}$ and $G_{2}$ ). Let $G^{\prime}$ be the bipartite graph $G^{\prime}$ obtained from $G$ by replacing each occurrence of $u$ in an edge of $G_{1}$ with $v$ and each occurrence of $v$ in an edge of $G_{1}$ with $u$ (the neighbors of $u$ and $v$ in $G_{1}$ are interchanged). Then $G^{\prime}$ is a bipartite graph with parts $Y_{1} \cup\left(X_{2} \backslash\{u\}\right)$ and $X_{1} \cup\left(Y_{2} \backslash\{v\}\right)$. We say that the graph $G^{\prime}$ is obtained from $G$ by a bi-twist with respect to the vertices $u$ and $v$, and $G^{\prime}$ is a bi-twist of $G$ (again with respect to vertices $u$ and $v$ ). It is easy to verify that bi-twists preserve both cycles and perfect matchings [2].

In the language of matrices, a bi-twist is described as follows. Let $A$ be the labeled $b i$-adjacency of order $n$ of $G$. By this we mean that the 1's of the ordinary bi-adjacency matrix (the 1's correspond to the edges of $G$ ) are replaced by distinct elements of some set. Since $\{u, v\}$ is a vertex cut of $G$, we may choose an ordering for the rows and columns of $A$, with $u$ corresponding to the first row and $v$ corresponding to the first column, so 
that $A$ has the form

$\left[\begin{array}{c|c|c}* & \alpha & \beta \\ \hline \gamma & A_{1} & O \\ \hline \delta & O & A_{2}\end{array}\right]$,

where

$\left[\begin{array}{c|c}* & \alpha \\ \hline \gamma & A_{1}\end{array}\right]$ and (ii) $\left[\begin{array}{l|l}* & \beta \\ \hline \delta & A_{2}\end{array}\right]$

are labeled bi-adjacency matrices of $G_{1}$ and $G_{2}$, respectively. Since $G$ is 1-extendable and so has a perfect matching, the matrices $A_{1}$ and $A_{2}$ are square. A labeled bi-adjacency matrix of $G^{\prime}$ is the matrix obtained from (1) by replacing (2)(i) with its transpose

$$
\left[\begin{array}{l|l}
* & \gamma^{T} \\
\hline \alpha^{T} & A_{1}^{T}
\end{array}\right]
$$

resulting in the matrix

$$
\left[\begin{array}{c|c|c}
* & \gamma^{T} & \beta \\
\hline \alpha^{T} & A_{1}^{T} & O \\
\hline \delta & O & A_{2}
\end{array}\right] .
$$

This matrix operation is called partial transposition in [1] and [2]. It follows from (1) that in order that a bipartite graph with parts of size $n$ have a bi-twist, its (labeled) adjacency matrix must have a $p$ by $q$ zero submatrix and a complementary $q$ by $p$ zero submatrix for some positive integers $p$ and $q$ with $p+q=n-1$.

In the language of bipartite graphs, the conjecture in [1] and [2] can be stated as follows.

Conjecture 1.1 Let $G$ and $G^{\prime}$ be two 1-extendable, bipartite graphs and let $\psi: E(G) \rightarrow$ $E\left(G^{\prime}\right)$ be a matching preserver. Then there is a sequence of bi-twists of $G$ resulting in a graph isomorphic to $G^{\prime}$ and $\psi$ is induced by this isomorphism.

As bi-twists do not suffice to describe all matching preservers between bipartite graphs, this conjecture is not true. 
Example 1.2 Let $G$ be the bipartite graph with labeled bi-adjacency matrix

$$
A=\left[\begin{array}{llllll}
a & b & c & 0 & 0 & 0 \\
d & e & f & 0 & 0 & 0 \\
0 & 0 & k & l & m & 0 \\
0 & 0 & r & s & t & 0 \\
w & 0 & 0 & 0 & u & v \\
z & 0 & 0 & 0 & x & y
\end{array}\right]
$$

with parts of size $n=6$. No bi-twist of $G$ (partial transposition of $A$ ) is possible. Yet the bipartite graph $G^{\prime}$ with labeled bi-adjacency matrix

$$
B=\left[\begin{array}{cccccc}
a & b & c & 0 & 0 & 0 \\
d & e & f & 0 & 0 & 0 \\
0 & 0 & u & v & w & 0 \\
0 & 0 & x & y & z & 0 \\
m & 0 & 0 & 0 & k & l \\
t & 0 & 0 & 0 & r & s
\end{array}\right]
$$

has the same collection of matchings as $G$. In fact, in both cases, the set of matchings is the union of the sets of matchings corresponding to the two labeled adjacency matrices

$$
\left[\begin{array}{cccccc}
a & b & 0 & 0 & 0 & 0 \\
d & e & 0 & 0 & 0 & 0 \\
0 & 0 & k & l & 0 & 0 \\
0 & 0 & r & s & 0 & 0 \\
0 & 0 & 0 & 0 & u & v \\
0 & 0 & 0 & 0 & x & y
\end{array}\right] \text { and }\left[\begin{array}{cccccc}
0 & b & c & 0 & 0 & 0 \\
0 & e & f & 0 & 0 & 0 \\
0 & 0 & 0 & l & m & 0 \\
0 & 0 & 0 & s & t & 0 \\
w & 0 & 0 & 0 & 0 & v \\
z & 0 & 0 & 0 & 0 & y
\end{array}\right]
$$

The operation in Example 1.2 in going from $G$ to $G^{\prime}$ is an instance of what we call bi-transposition and which we now define. It is the only other operation in addition to bi-twists that is needed in order to describe matching preservers.

Let $G_{1}, G_{2}, G_{3}$ be bipartite graphs with bipartitions $\left(V_{1}^{i}, V_{2}^{i}\right)$, and having pairwise disjoint vertex sets. We further assume that $\left|V_{1}^{i}\right|=\left|V_{2}^{i}\right|+1$. Let $a_{i}, b_{i}$ be vertices from the part $V_{1}^{i}$ of $G_{i}, i=1,2,3$. Let $G$ be the bipartite graph obtained from $G_{1}, G_{2}, G_{3}$ by identifying the vertices in each of the three pairs $\left\{b_{1}, a_{2}\right\},\left\{b_{2}, a_{3}\right\}$, and $\left\{b_{3}, a_{1}\right\}$. Let $G^{\prime}$ be the bipartite graph obtained from $G_{1}, G_{2}, G_{3}$ by identifying the vertices in each of the three pairs $\left\{b_{1}, a_{3}\right\},\left\{b_{2}, a_{1}\right\}$, and $\left\{b_{3}, a_{2}\right\}$. Then graph $G^{\prime}$ is said to be obtained from $G$ by a bi-transposition of $G_{1}, G_{2}$ and $G_{3}$ (see Figure 1 ).

It is straightforward to verify that the operation of bi-transposition also preserves both cycles and perfect matchings, but cannot be replaced by the bi-twists. The following is the main result of this paper. It is proved in the last section.

Theorem Let $G$ and $G^{\prime}$ be two 1-extendable, bipartite graphs and let $\psi: E(G) \rightarrow E\left(G^{\prime}\right)$ be a matching preserver. Then there is a sequence of bi-twists and bi-transpositions of $G$ resulting in a graph isomorphic to $G^{\prime}$ and $\psi$ is induced by this isomorphism. 

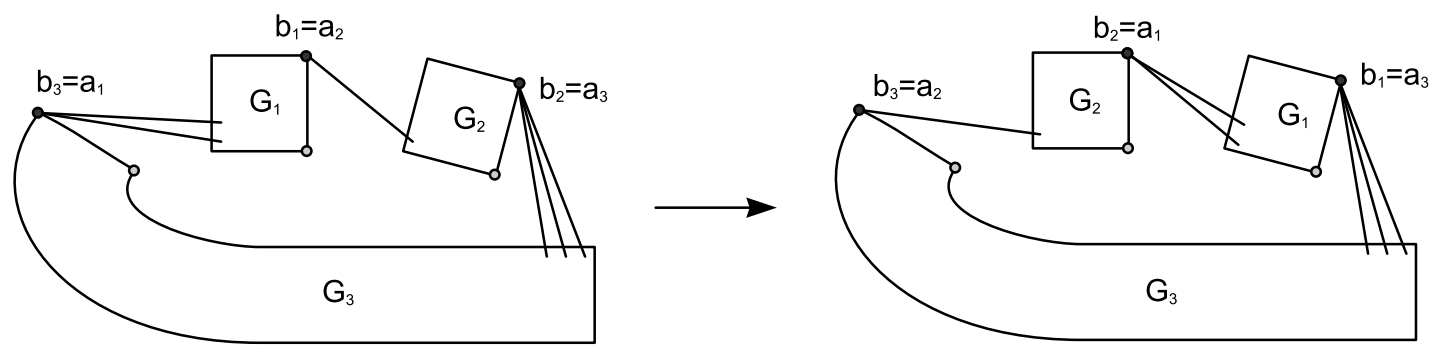

Figure 1: Bi-transposition.

\section{Preliminaries}

In this section we review some facts that will be used in our proof of Theorem 4.1. Let $G$ and $G^{\prime}$ be 1-extendable bipartite graphs, and suppose that $\psi: E(G) \rightarrow E\left(G^{\prime}\right)$ is a matching preserver. By Theorem 2.4 of [2], and it is not difficult to prove, there is a bijection between the components of $G$ and $G^{\prime}$ such that $\psi$ induces a matching preserver between corresponding components. Hence we may restrict our attention to connected, 1-extendable bipartite graphs - in matrix terms, to fully indecomposable matrices.

The next lemma follows from the inductive structure of a nearly decomposable matrix (see [3]), equivalently from the ear structure of elementary bipartite graphs (see [4]). For convenience, we give a short self-contained proof.

Lemma 2.1 Let $G=(V, E)$ be a 1-extendable, connected bipartite graph. Then $G$ has a perfect matching $M$ such that for each edge e of $M$, the vertices of e do not form a cut in $G$.

Proof: It suffices to show that if $\{u, v\}$ is a vertex cut such that $e=u v$ is an edge, then the graph $G \backslash e$ obtained from $G$ by deleting edge $e$ is 1-extendable and connected. By recursively deleting such edges we arrive at a 1-extendable, connected bipartite graph $G^{\prime}$, where $G^{\prime}$ has a perfect matching $M$ and for all edges $e^{\prime}=u^{\prime} v^{\prime}$ of $G^{\prime}$, in particular for those in $M,\left\{u^{\prime}, v^{\prime}\right\}$ is not a cut of $G^{\prime}$ and hence not a cut of $G$.

Since 1-extendable, connected graphs are always 2-connected, it suffices to show that $G \backslash e$ is 1-extendable. There are subgraphs $G_{1}$ and $G_{2}$ such that $V\left(G_{1}\right) \cup V\left(G_{2}\right)=V(G)$, $V\left(G_{1}\right) \cap V\left(G_{2}\right)=\{u, v\}, E\left(G_{1}\right) \cup E\left(G_{2}\right)=E(G)$ and $E\left(G_{1}\right) \cap E\left(G_{2}\right)=\{e\}$. Each perfect matching not containing $e$ has both of the edges incident with $\{u, v\}$ contained in the same $G_{i}$.

Let $e^{\prime}$ be an edge of $G \backslash e$. Let $M^{\prime}$ be a perfect matching of $G$ containing $e^{\prime}$. If $e \notin M^{\prime}$, then $M^{\prime}$ is also a perfect matching of $G^{\prime}$ and contains $e^{\prime}$. Assume that $e \in M^{\prime}$, and that e.g. $e^{\prime}$ is an edge of $G_{1}$. Let $M^{\prime \prime}$ be the restriction of $M^{\prime}$ to a perfect matching of $G_{1}$. Let $f \neq e$ be an edge of $G_{2}$ incident with $\{u, v\}$ and let $N$ be a perfect matching of $G$ containing $f$. Then $N$ contains a perfect matching $N_{2}$ of $G_{2}$. Thus $\left(M^{\prime \prime} \backslash\{e\}\right) \cup N_{2}$ is a perfect matching of $G \backslash e$ containing $e^{\prime}$, and this completes the proof. 
We now review a classical theorem of Whitney [8]. Let $G$ be a 2-connected graph with vertex cut $\{u, v\}$. There are subgraphs $G_{1}$ and $G_{2}$ such that $V\left(G_{1}\right) \cup V\left(G_{2}\right)=V(G)$, $V\left(G_{1}\right) \cap V\left(G_{2}\right)=\{u, v\}, E\left(G_{1}\right) \cup E\left(G_{2}\right)=E(G)$ and $E\left(G_{1}\right) \cap E\left(G_{2}\right)=\{u v\}$ or $\emptyset$ depending on whether or not $u v$ is an edge of $G$. Define a graph $G^{\prime}$ as follows: Let $G^{\prime}$ be the graph obtained from $G$ by replacing each occurrence of $u$ in an edge of $G_{1}$ with $v$ and each occurrence of $v$ in an edge of $G_{1}$ with $u$ (the neighbors of $u$ and $v$ in $G_{1}$ are interchanged). Then $G^{\prime}$ is obtained from $G$ by a twist, and $G^{\prime}$ is a twist of $G$ (again with respect to vertices $u$ and $v$ ). (If $u v$ is an edge of $G$, then it is also an edge of $G^{\prime}$.) It was proved by Whitney [8] that each graph with the same cycles as the 2-connected graph $G$-that is, a graph that is 2-isomorphic to $G$-can be obtained from $G$ by a sequence of twists. Truemper [6] simplified the proof and obtained a bound on the number of twists needed.

Theorem 2.2 Let $G$ be a 2-connected graph with $n \geq 2$ vertices, and let $H$ be a graph 2 -isomorphic to $G$. Then $G$ can be transformed into a graph $G^{*}$ which is isomorphic to $H$ by a sequence of at most $n-2$ twists.

The technique of Truemper uses the concept of generalized cycles. A graph $G$ is a generalized cycle with constituents $G_{1}, G_{2}, \ldots, G_{k}(k \geq 2)$ provided that the following hold:

(i) each $G_{i}$ is a connected subgraph of $G$ having nonempty edge set $E_{i}$; additionally, if $k=2$ then both $G_{1}$ and $G_{2}$ contain at least three vertices;

(ii) the edge sets $E_{i}, 1 \leq i \leq k$, partition the edge set $E(G)$, and each $G_{i}$ has exactly two vertices in common with $\cup_{j \neq i} G_{j}$ (these vertices are called the contact vertices of $\left.G_{i}\right)$;

(iii) replacing each $G_{i}$ by an edge joining the contact vertices of $G_{i}$ produces an ordinary cycle.

The generalized cycle $G$ is a connected graph. If $k \geq 3$ and each $G_{i}$ has only two vertices (since $G_{i}$ is connected, these two vertices are joined by an edge), then $G$ is an ordinary cycle.

The first assertion in the next lemma is due to Tutte [7]; the second assertion is due to Truemper [6]. In the lemma, a $G_{i}$ consisting of a single edge is regarded as 2-connected.

Lemma 2.3 If a graph $G$ is 2-connected but not 3-connected, then there exists a representation of $G$ as a generalized cycle where each constituent is 2-connected.

Moreover, let $G$ be a 2-connected, generalized cycle as above. If $\psi: E(G) \rightarrow E(H)$ is a 2-isomorphism of $G$ to $H$, then $H$ is a generalized cycle with constituents $H_{1}, H_{2}, \ldots, H_{k}$, where $H_{i}$ is the subgraph of $H$ induced by $\psi\left(E_{i}\right)$ for $1 \leq i \leq k$. 


\section{$3 \quad$ Directed graphs}

There is a well-known correspondence between matchings in a bipartite graph $G$ and circuits in a directed graph (digraph) $D$ constructed from $G$ and a specified perfect matching of $G$. This correspondence can be easily understood by using adjacency matrices. Let $M=\left\{u_{1} v_{1}, u_{2} v_{2}, \ldots, u_{n} v_{n}\right\}$ be a perfect matching of $G$ and let $A=\left[a_{i j}\right]$ be the biadjacency matrix of $G$ where $a_{i j}=1$ if and only if $u_{i} v_{j}$ is an edge of $G, 1 \leq i, j \leq n$. Thus $A$ has all 1's on its main diagonal and these 1's correspond to the edges of $M$. The matrix $A-I_{n}$ is the adjacency matrix of a digraph $D(G, M)$. The digraph can also be understood as obtained from $G$ by orienting each edge from one part of $G$ to its other part,and then contracting all of the edges of $M$.

A circuit of a digraph is a circular sequence of distinct edges such that the terminal vertex of each edge is the initial vertex of the edge that follows. As such, a circuit may be identified with its collection of edges, since its circular arrangement is unique. Similarly, we may identify a path in a digraph with its collection of edges.

Let $M^{\prime}$ be another perfect matching in $G$. Then $\left(M \backslash M^{\prime}\right) \cup\left(M^{\prime} \backslash M\right)$ is a collection of pairwise vertex disjoint cycles of $G$ of even length whose edges alternate between $M$ and $M^{\prime}$. In $D(G, M)$ these cycles correspond to pairwise vertex-disjoint circuits (not necessarily a spanning set since $M$ and $M^{\prime}$ may have edges in common). Using the matching $M$, we may reverse this construction to obtain, given a collection of pairwise vertex-disjoint circuits of $D(G, M)$, a perfect matching $M^{\prime}$ of $G$. Thus, there is a oneto-one correspondence between perfect matchings in $G$ and collections of pairwise-vertex disjoint circuits in $D(G, M)$. This well-known observation allows us to reformulate our problem in terms of digraphs and pairwise vertex-disjoint circuits.

A digraph is strongly connected provided that for each ordered pair of vertices $u, v$, there is a path from $u$ to $v$. The 1-extendability of the connected bipartite graph is equivalent to the strong connectivity of $D(G, M)$. We formalize this well-known property in the next lemma (see e.g. [3]). (In matrix terms this property is usually stated as: A $(0,1)$-matrix $A$ of order $n$ with all 1's on its main diagonal is fully indecomposable if and only if the matrix $A-I_{n}$ is irreducible.

Lemma 3.1 Let $G$ be a connected bipartite graph and let $M$ be a perfect matching of $G$. Then $G$ is 1-extendable if and only if the digraph $D(G, M)$ is strongly connected.

The analogue of Whitney's theorem for digraphs was proved by Thomassen [5]. First, recall that an isomorphism, respectively, an anti-isomorphism, of a digraph $D$ onto a digraph $D^{\prime}$ is a bijection $f: V(D) \rightarrow V\left(D^{\prime}\right)$ such that, for all $u, v \in V(D)$, there is an arc in $D$ from vertex $u$ to vertex $v$ if and only if there is an arc in $D^{\prime}$ from vertex $f(u)$ to vertex $f(v)$, respectively, from $f(v)$ to $f(u)$.

A directed twist of a digraph $D$ is defined in a similar way to a twist in a graph. Let $D_{1}, D_{2}$ be subgraphs of $D$ of order at least 3 , such that $V\left(D_{1}\right) \cup V\left(D_{2}\right)=V(D)$, $V\left(D_{1}\right) \cap V\left(D_{2}\right)=\{u, v\}, E\left(D_{1}\right) \cup E\left(D_{2}\right)=E(D)$. Let $D^{\prime}$ be obtained from $D$ by replacing arcs of the form $u w, w u, v w$, and $w v$ by, respectively, $w v, v w, w u$ and $u w$ for each $w \in V\left(D_{2}\right)$ and then reversing the direction of all the remaining arcs of $D_{2}$. Then 
$D^{\prime}$ is obtained from $D$ by a directed twist (or di-twist), with respect to the vertices $u$ and $v$, and $D^{\prime}$ is a di-twist of $D$ (again with respect to the vertices $u$ and $v$ ). Clearly, $D$ and $D^{\prime}$ have the same circuits and $D$ is strongly connected if and only if $D^{\prime}$ is.

In the language of matrices, a directed twist is described as follows. Let $A$ be the adjacency matrix of the digraph $D$ where the vertices have been ordered so that $u$ and $v$ come first followed by the remaining vertices of $D_{1}$ and then the remaining vertices of $D_{2}$. Thus $A$ has the form

$\left[\begin{array}{c|c|c|c}a & b & \alpha_{1} & \alpha_{2} \\ \hline c & d & \beta_{1} & \beta_{2} \\ \hline \gamma_{1} & \delta_{1} & A_{1} & O \\ \hline \gamma_{2} & \delta_{2} & O & A_{2}\end{array}\right]$,

where

(i)

$\left[\begin{array}{c|c|c}a & b & \alpha_{1} \\ \hline c & d & \beta_{1} \\ \hline \gamma_{1} & \delta_{1} & A_{1}\end{array}\right]$

and (ii)

$\left[\begin{array}{c|c|c}a & b & \alpha_{2} \\ \hline c & d & \beta_{2} \\ \hline \gamma_{2} & \delta_{2} & A_{2}\end{array}\right]$

are the adjacency matrices of $D_{1}$ and $D_{2}$, respectively. An adjacency matrix of the digraph $D^{\prime}$ is obtained from (5) by replacing (6)(ii) in (5) with

$\left[\begin{array}{c|c|c}a & c & \delta_{2}^{T} \\ \hline b & d & \gamma_{2}^{T} \\ \hline \beta_{2}^{T} & \alpha_{2}^{T} & A_{2}^{T}\end{array}\right]$

Thomassen [5] proved a analogue of Whitney's theorem for digraphs, applying Whitney's theorem to the underlying graph. If $D$ is a digraph, then $G_{D}$ denotes the underlying graph of $G$.

Theorem 3.2 Let $D$ and $D^{\prime}$ be two strongly connected digraphs with 2-connected underlying graphs $G_{D}$ and $G_{D^{\prime}}$. Let $\varphi: E(D) \rightarrow E\left(D^{\prime}\right)$ be a bijection such that $\varphi$ and $\varphi^{-1}$ preserve circuits. Then there exist a sequence of di-twists of $D$ resulting in a digraph $D^{*}$ such that $\varphi$ is induced by an isomorphism or anti-isomorphism of $D^{*}$ onto $D^{\prime}$.

Note that the requirement of the 2-connectivity of the underlying graphs is necessary only for $G_{D}$. That $G_{D^{\prime}}$ is 2-connected then follows from Whitney's theorem.

Let $D$ be a digraph. Then $D$ is a generalized circuit provided $D$ is strongly connected and the underlying graph $G_{D}$ is a generalized cycle. Let $D$ be a generalized circuit such that the constituents of its underlying graph $G$ are $G_{1}, G_{2}, \ldots, G_{k}$, and the contact vertices 
in $G_{i}$ are $u_{i}$ and $v_{i}, i=1,2, \ldots, k$. Then the corresponding digraphs $D_{1}, D_{2}, \ldots, D_{k}$ are called the constituents of the generalized circuit $D$, and the vertices $u_{i}, v_{i}$ in $D_{i}$ are called its contact vertices, $i=1,2, \ldots, k$. Note that $v_{i}=u_{i+1}$ where the subscripts are interpreted modulo $k$. Moreover, in the rest of the paper, we work only with generalized circuits with the underlying graphs of all the constituents 2-connected.

Since $D$ is assumed to be strongly connected, it follows that, for each constituent $D_{i}$, either $D_{i}$ is strongly connected or the digraph obtained from $D_{i}$ by contracting each strong component to a vertex contains a path with initial vertex corresponding to the strong component containing $u_{i}$ and final vertex corresponding to the strong component containing $v_{i}$, or the other way around. The following lemma is now easily verified.

Lemma 3.3 If $D$ is a generalized circuit, then $D$ has a circuit $C$ containing all of the contact vertices, and passing through all of its constituents.

In a generalized circuit $D$, we always assume that its constituents have been labeled $D_{1}, D_{2}, \ldots, D_{k}$ in such a way that the circuit $C$ in Lemma 3.3 comes into $D_{i}$ at $u_{i}\left(=v_{i-1}\right)$ and leaves $D_{i}$ at $v_{i}\left(=u_{i+1}\right)$.

Now we state a directed analogue of the second assertion in Lemma 2.3.

Lemma 3.4 Let $D$ be a generalized circuit with constituents $D_{1}, D_{2}, \ldots, D_{k}$ and let the underlying graphs of all $D_{i}$ be 2-connected, $i=1,2, \ldots k$. Let $E_{i}$ denote the edge-set of $D_{i}$. Let $\varphi: E(D) \rightarrow E\left(D^{\prime}\right)$ be a bijection such that $\varphi$ and $\varphi^{-1}$ preserve circuits. Then $D^{\prime}$ is a generalized circuit with constituents $D_{1}^{\prime}, D_{2}^{\prime}, \ldots, D_{k}^{\prime}$ (not necessarily ordered in this way), where $D_{i}^{\prime}$ is the subgraph of $D^{\prime}$ induced by $\varphi\left(E_{i}\right)$ for $1 \leq i \leq k$.

Proof: The corresponding underlying graphs $G_{D}$ and $G_{D^{\prime}}$ are 2-isomorphic by Theorem 3.2. The lemma now follows by applying Lemma 2.3.

The contact vertices of the generalized circuit $D$ are partitioned into three types. If there is a circuit in $D_{i}$ meeting one of its contact vertices $u_{i}, v_{i}$ but not the other, then we say that that contact vertex is heavy in $D_{i}$. If there is no circuit in $D_{i}$ containing a particular contact vertex, then we call that contact vertex light in $D_{i}$; in this case, the strong component of $D_{i}$ containing the contact vertex contains no other vertex. If a contact vertex in $D_{i}$ is neither light nor heavy, then it is called cyclic in $D_{i}$; if e.g. $u_{i}$ is cyclic in $D_{i}$, then there is a circuit containing $u_{i}$, and each circuit in $D_{i}$ containing $u_{i}$ also contains $v_{i}$. Note that if one of the contact vertices is cyclic in $D_{i}$, then the second one clearly cannot be light.

Let $D$ be a generalized circuit with constituents $D_{i}$ (each of them has 2-connected underlying graph) and contact vertices $u_{i}, v_{i}, i=1,2, \ldots, k$. Let $\sigma$ be a permutation of $\{1,2, \ldots, k\}$ and let $\varepsilon \in\{-1,+1\}^{k}$. Let the digraph $D^{\sigma, \varepsilon}$ be obtained from $D$ by rearranging the constituents in the following way: First, assume a directed graph consisting of disjoint components $D_{1}, D_{2}, \ldots D_{k}$. Then, for $i=1,2, \ldots k$, if $\varepsilon_{i}=-1$ reverse the orientation of the edges of $D_{i}$ and set $x_{i}=v_{i}, y_{i}=u_{i}$; if $\varepsilon=+1$ set $x_{i}=u_{i}$ and $y_{i}=v_{i}$. 
Finally, we identify vertices $y_{\sigma(i)}$ and $x_{\sigma(i+1)}$ (modulo $k$ ), for $i=1,2, \ldots, k$. Moreover, we call the rearrangement $D^{\sigma, \varepsilon}$ an admissible rearrangement of $D$ if the following property holds:

(*) If $v_{i}$ is heavy or cyclic in $D_{i}$ and $u_{i+1}$ is heavy or cyclic in $D_{i+1}$, then $D_{i+1}$ follows $D_{i}$ in $D^{\sigma, \varepsilon}$ as it does in $D$ or $D_{i}$ follows $D_{i+1}, i=1,2, \ldots, k$. If, in addition, $v_{i}\left(u_{i+1}\right.$, respectively) is actually heavy in $D_{i}\left(D_{i+1}\right.$, respectively) then $v_{i}\left(u_{i+1}\right.$, respectively) is one of the contact vertices of $D_{i}$ and $D_{i+1}$ in $D^{\sigma, \varepsilon}$.

It follows, in particular, that an admissible rearrangement of a circuit produces either the circuit itself or its reversal (as, by our convention, a set of edges).

The definition of an admissible rearrangement defines implicitly a partition of the constituents of $D$ into superconstituents, where each superconstituent is a maximal sequence of consecutive constituents of $D$ with the property that no inner contact vertex is light. It follows that a common contact vertex of two different superconstituents is light in at least one of them.

It is straightforward to verify the following lemma.

Lemma 3.5 Let $D$ be a generalized circuit. A rearrangement of the constituents of $D$ does not change the set of circuits incident to any vertex of $D$ if and only if it is an admissible rearrangement.

The following theorem is a first step towards characterizing bijections between the edges of two digraphs that preserve the union of vertex-disjoint circuits.

Theorem 3.6 Let $D$ and $D^{\prime}$ be two strongly connected digraphs and let $\varphi: E(D) \rightarrow$ $E\left(D^{\prime}\right)$ be a bijection. Then $\varphi$ induces a bijection between unions of vertex-disjoint circuits of $D$ and unions of vertex-disjoint circuits of $D^{\prime}$ (that is, $\varphi$ and $\varphi^{-1}$ preserve unions of vertex-disjoint circuits) if and only if, starting with $D$, there is a sequence of admissible rearrangements of generalized circuits that results in a digraph $D^{\dagger}$ such that $\varphi$ is induced by an isomorphism or anti-isomorphism of $D^{\dagger}$ onto $D^{\prime}$.

Proof: It follows from Lemma 3.5 that admissible rearrangements do not change the set of vertex-disjoint circuits. We now consider the converse. First we note that the converse holds when $D$ (and hence $D^{\prime}$ ) is a circuit. Let $D$ be a minimal counterexample, and let $\Gamma$ be a generalized circuit of $D$ with the underlying graphs of all its constituents 2-connected; $\Gamma$ exists by Lemma 2.3. Lemma 3.4 implies that the image $\Gamma^{\prime}$ of $\Gamma$ under $\varphi$ is a generalized circuit of $D^{\prime}$ whose constituents $\Gamma_{i}^{\prime}$ are images under $\varphi$ of the constituents $\Gamma_{i}$ of $\Gamma$. Let $\Gamma_{i}^{*}$ be the strongly connected digraph obtained from $\Gamma_{i}$ by adding a new edge from its contact vertex $v_{i}$ to its contact vertex $u_{i}$, and let $\Gamma_{i}^{* *}$ be defined in a similar way using contact vertices $u_{i}^{\prime}$ and $v_{i}^{\prime}$. Let $\varphi_{i}^{*}$ be the extension of $\varphi \mid \Gamma_{i}$ to $\Gamma_{i}^{*}$ which maps the edge $\left(v_{i}, u_{i}\right)$ to the edge $\left(v_{i}^{\prime}, u_{i}^{\prime}\right)$. In particular, $\varphi_{i}^{*}$ is a bijection from the edges of $\Gamma_{i}^{*}$ to those of $\Gamma_{i}^{* *}$ preserving the set of unions of vertex-disjoint circuits of $\Gamma_{i}^{*}$. Hence, by minimality of $D$, each $\varphi_{i}^{*}$ is realized by admissible rearrangements of constituents of generalized circuits of $\Gamma_{i}^{*}$. Each admissible rearrangement of $\Gamma_{i}^{*}$ corresponds to an admissible rearrangement of $\Gamma$. It follows that the generalized circuit $\Gamma^{\prime}$ is obtained by a rearrangement of the 
constituents of the generalized circuit whose constituents are admissible rearrangements of the constituents of $\Gamma$. By Lemma 3.5, this rearrangement must be admissible, since a non-admissible rearrangement would change the set of unions of vertex-disjoint circuits.

By Theorem 3.6, to fully characterize mappings that preserve unions of vertex-disjoint circuits, it suffices to describe how admissible rearrangements can be carried out. To do this, we introduce two operations, that of a cyclic di-twist and of a supertransposition.

A di-twist of a strongly connected digraph $D$ with parts $D_{1}$ and $D_{2}$ is cyclic if both twist vertices are light in one of the parts, say in $D_{1}$ (here we regard $D$ as a generalized circuit with two constituents each with at least three vertices). What this means is the following: Let $V\left(D_{1}\right) \cap V\left(D_{2}\right)=\{u, v\}$ where $u$ and $v$ are light in $D_{1}$. Then $\{u\}$ and $\{v\}$ are strong components of $D_{1}$ with, say, each edge of $D_{1}$ incident with $u$ directed from $u$ and each edge of $D_{1}$ incident with $v$ directed to $v$. Then the di-twist with respect to the parts $D_{1}$ and $D_{2}$ is a cyclic di-twist. It follows directly that a cyclic di-twist of a digraph preserves unions of vertex-disjoint circuits.

Now let $D$ be a generalized circuit. Let $S$ and $S^{\prime}$ be consecutive superconstituents and let $u$ and $v$ (respectively, $u^{\prime}$ and $v^{\prime}$ ) be the contact vertices of $S$ (respectively, of $S^{\prime}$ ), where $u^{\prime}=v$. Further, let $T$ be the union of all remaining superconstituents with contact vertices $u_{T}$ and $v_{T}$ where $u_{T}=v^{\prime}$ and $v_{T}=u$. Assume that $u, u^{\prime}$, and $u_{T}$ are light in, respectively, $S, S^{\prime}$, and $T$. Then the operation of supertransposition of superconstituents $S$ and $S^{\prime}$ results in the digraph $D^{\prime}$ constructed by transposing $S$ and $S^{\prime}$ in the following way: $D^{\prime}$ is obtained from the disjoint union of $S, S^{\prime}$, and $T$ by identifying $u$ with $v^{\prime}, u^{\prime}$ with $v_{T}$ and $u_{T}$ with $v$. It is straightforward to verify that supertransposition does not change vertex-disjoint unions of circuits. Our goal is now to show that cyclic di-twists and supertransposition can be used to completely describe edge bijections between strongly connected digraphs which preserve vertex-disjoint unions of circuits.

Consider a generalized circuit $D$ with constituents $D_{1}, \ldots, D_{k}$ and superconstituents $S_{1}, S_{2}, \ldots, S_{l}$. We associate with $D$ an object called an auxiliary circuit that captures the relationship between the superconstituents. The auxiliary circuit has length $l$ and an edge $e_{i}$, joining the contact vertices $u_{i}, v_{i}$ of $S_{i}, i=1,2, \ldots, l$; thus, each superconstituent is contracted to an edge joining its two contact vertices, and these edges are of three types. Edge $e_{i}$ is a fat-edge provided that neither $u_{i}$ nor $v_{i}$ is a light vertex of $S_{i}$ (fat-edges have no direction); $e_{i}$ is a thin-edge and is directed from $u_{i}$ to $v_{i}$ provided that $v_{i}$ is a light vertex of $S_{i}$ while $u_{i}$ is not: $e_{i}$ is a two-way edge directed to both $u_{i}$ and $v_{i}$ provided both $u_{i}$ and $v_{i}$ are light vertices of $S_{i}$. Important properties of the auxiliary circuit, following from the definition of superconstituents, are:

AC1. There is at least one edge directed to each of the vertices of an auxiliary circuit. Hence:

AC2. If an auxiliary circuit has at least one fat-edge, then it has a two-way edge.

AC3. If an auxiliary circuit contains no two-way edges, then it is just an ordinary directed circuit. 
We now define admissible rearrangements for auxiliary circuits. A two-way path is a path of the auxiliary circuit which starts and ends with a two-way edge. An admissible rearrangement of the edges of an auxiliary circuit $C$ is an auxiliary circuit $C^{\prime}$ obtained by arbitrarily rearranging the fat-edges amongst themselves, arbitrarily rearranging the two-way paths amongst themselves, and removing thin-edges from their places and reinserting them in other places with orientations reversed (with respect to the clockwise orientation). Note that fat-edge rearrangements and two-way path rearrangements applied to an auxiliary circuit always result in an auxiliary circuit; a thin-edge shift requires that the property that at least one edge is directed to each of the vertices be maintained. The next lemma shows that two auxiliary circuits, with the same number of edges of each of the three types and with at least one two-way edge, are admissible rearrangements of one another.

Lemma 3.7 Let $C$ and $C^{\prime}$ be auxiliary circuits containing the same number of edges of each type, with at least one two-way edge. For $C$ and $C^{\prime}$, let there be assigned labels so that for each of the three types of edges, the edges have the same set of labels in $C$ as in $C^{\prime}$. Then $C^{\prime}$ can be obtained from $C$ by fat-edge rearrangements, two-way path rearrangements, and thin-edge shifts.

Proof: First we rearrange the labeled fat-edges and rearrange the labeled two-way paths of $C$ so that the fat edges and the two-way edges agree with their cyclic positioning in $C^{\prime}$. As remarked above, the result is an auxiliary circuit. Then by shifting the labeled thin-edges of $C$, we get them to be in the right position in $C^{\prime}$ : if the orientation of a thin-edge needs to be changed, then one shift is sufficient; otherwise, we shift the thin edge next to a two-way edge, thereby changing its orientation and then shift it to its right place, thereby changing its orientation back to what it was.

We next consider how a cyclic di-twist of a generalized circuit $D$ relates to the corresponding auxiliary circuit $C$. Let $T_{1}$ and $T_{2}$ be a partition of the superconstituents of $D$ into two consecutive parts, where $V\left(T_{1}\right) \cap V\left(T_{2}\right)=\{u, v\}$. The di-twist with respect to $T_{1}$ and $T_{2}$ is cyclic provided that $u$ and $v$ are light in one of the parts, say $T_{1}$. Denote by $P_{i}$ the set of edges of the auxiliary circuit corresponding to the superconstituents in $T_{i}, i=1,2$. Then $P_{1}$ and $P_{2}$ partition the edges of the auxiliary circuit into two paths (consisting of the three types of edges). Since $u$ and $v$ are light in $T_{1}$, the edge of $P_{1}$ at $u$, respectively, $v$ is either a two-way edge or a thin edge directed to $u$, respectively, to $v$. Conversely, when we have such a partition of the auxiliary circuit with these properties, the di-twist with respect to corresponding parts of $D$ is cyclic.

Lemma 3.8 Let $C$ and $C^{\prime}$ be auxiliary circuits containing the same number of edges of each type, with at least one two-way edge. For $C$ and $C^{\prime}$, let there be assigned labels so that for each of the three types of edges, the edges have the same set of labels in $C$ as in $C^{\prime}$. Then $C^{\prime}$ can be obtained from $C$ by a sequence of cyclic di-twists. 
Proof: First consider an exchange of two fat-edges edges $e_{i}$ and $e_{j}$. Let $S_{i}$ and $S_{j}$ be corresponding superconstituents. Since no fat-edges are consecutive in $C, C \backslash\left\{e_{i}, e_{j}\right\}$ consists of two paths $P_{1}$ and $P_{2}$. Denote by $T_{m}$ the union of superconstituents corresponding to edges of $P_{m}, m=1,2$. Then the exchange of $e_{i}$ and $e_{j}$ can be constructed by two cyclic di-twists: first twist with parts $T_{1}$ and $S_{i} \cup T_{2} \cup S_{j}$ and then twist with parts $T_{2}$ and $S_{i} \cup T_{1} \cup S_{j}$.

Now consider an exchange of two two-way paths $e_{i}$ and $e_{j}$. Their exchange by cyclic di-twists is similar to that for fat-edges. Let $S_{i}$ and $S_{j}$ be the corresponding superconstituents. First suppose that the two-way paths $e_{i}, e_{j}$ are consecutive so that $P=C \backslash\left\{e_{i}, e_{j}\right\}$ is a path. Denote by $T$ the union of the corresponding superconstituents. Then the exchange of $e_{i}$ and $e_{j}$ can be carried out by the cyclic di-twist with parts $T$ and $S_{i} \cup S_{j}$. Now suppose that $e_{i}, e_{j}$ are not consecutive. Let $P_{1}$ and $P_{2}$ be the two paths of $C \backslash\left\{e_{i}^{\prime}, e_{j}^{\prime}\right\}$, and let $T_{1}$ and $T_{2}$ be the corresponding unions of superconstituents. Then the exchange of $e_{i}$ and $e_{j}$ can be carried out by two cyclic di-twists: first twist with parts $T_{1}$ and $S_{i} \cup T_{2} \cup S_{j}$ and then twist with parts $T_{2}$ and $S_{i} \cup T_{1} \cup S_{j}$.

Finally, we show how a thin-edge shift can be carried out by cyclic di-twists. Let $e$ be a thin-edge, and let $v$ be the vertex of $C$ (and so a contact vertex of the corresponding generalized circuit) to which $e$ is to be shifted. Let $S_{e}$ be the superconstituent corresponding to $e$. Finally, let $P_{1}$ and $P_{2}$ be the paths from $v$ to the closest vertex of $e$ in both directions of $C$, and let $T_{1}$ and $T_{2}$ be the corresponding unions of superconstituents. Then the shift of $e$ can be constructed by two cyclic di-twists: first twist with parts $T_{1}$ and $T_{2} \cup S_{e}$ and then twist with parts $T_{2}$ and $T_{1} \cup S_{e}$.

We now apply the previous two lemmas to admissible rearrangements of generalized circuits. An admissible rearrangement of a generalized circuit is an admissible superconstituent rearrangement provided that it only rearranges the superconstituents.

Lemma 3.9 Let $D$ be a generalized circuit. Then each admissible superconstituent rearrangement $D^{\prime}$ of $D$ can be accomplished by a sequence of cyclic di-twists and supertranspositions.

Proof: Let $C$ and $C^{\prime}$ be the auxiliary circuits corresponding to $D$ and $D^{\prime}$, respectively. Then the superconstituent rearrangement induces a bijection between the edges of $C$ and those of $C^{\prime}$ that preserves edge types. If the auxiliary circuit $C$ of $D$ contains at least one two-way edge, then by Lemma 3.7, $C^{\prime}$ may be obtained from $C$ by a sequence of fat-edge exchanges, two-way edge exchanges, and thin-edge shifts. By Lemma 3.8, this can be accomplished by a sequence of cyclic di-twists. Suppose that $C$ does not contain any two-way edges. Then $C$ is an ordinary circuit, and $C^{\prime}$ is also an ordinary circuit obtained by a rearrangement of the edges of $C$. But then $C^{\prime}$ can be obtained from $C$ by a sequence of transpositions of consecutive edges. Each such transposition of edges can be accomplished by a supertransposition. 
We now characterize bijections between the edges of digraphs that preserve unions of vertex-disjoint circuits.

Theorem 3.10 Let $D$ and $D^{\prime}$ be two strongly connected digraphs, and let $\varphi: E(D) \rightarrow$ $E\left(D^{\prime}\right)$ be a bijection. Then $\varphi$ induces a bijection between unions of vertex-disjoint circuits of $D$ and unions of vertex-disjoint circuits of $D^{\prime}$ if and only if, starting with $D$, there is a sequence of cyclic di-twists and supertranspositions that results in a digraph $D^{\dagger}$ such that $\varphi$ is induced by an isomorphism or anti-isomorphism of $D^{\dagger}$ onto $D^{\prime}$.

Proof: Each admissible rearrangement consists of an admissible rearrangement of superconstituents and admissible rearrangements within a superconstituent. Moreover, admissible rearrangements within a superconstituent can be accomplished by cyclic di-twists. Hence the theorem follows by Theorem 3.6 and Lemma 3.9.

\section{Perfect Matching Preservers}

We can now prove the main result of this paper.

Theorem 4.1 Let $G$ and $G^{\prime}$ be two 1-extendable, bipartite graphs and let $\psi: E(G) \rightarrow$ $E\left(G^{\prime}\right)$ be a matching preserver. Then there is a sequence of bi-twists and bi-transpositions of $G$ resulting in a graph isomorphic to $G^{\prime}$ and $\psi$ is induced by this isomorphism.

Proof: By Lemma 2.1, $G$ has a perfect matching $M$ with the property that the two vertices of each edge do not form a cut of $G$. A bi-twist of the bipartite graph $G$ corresponds to a cyclic twist of the digraph $D(G, M)$, and it follows from the definition of the supertransposition, in particular from the assumption of the lightness of the connecting vertices, that bi-transposition of $G$ corresponds to a supertransposition of $D(G, M)$. As observed previously, bi-twists and bi-transpositions preserve perfect matchings.

For the converse, assume that $\psi: E(G) \rightarrow E\left(G^{\prime}\right)$ is a matching preserver. Let $M^{\prime}=\psi(M)$. Let $\varphi: E(D(G, M)) \rightarrow E\left(D\left(G^{\prime}, M^{\prime}\right)\right)$ be the bijection naturally determined by $\psi$. From the correspondence between matchings in $G$, respectively, $G^{\prime}$, and unions of vertex-disjoint circuits of $D(G, M)$, respectively $D\left(G^{\prime}, M^{\prime}\right), \varphi$ induces a bijection between pairwise vertex-disjoint circuits of $D(G, M)$ and those of $D\left(G^{\prime}, M^{\prime}\right)$. Hence by Theorem 3.10, $D\left(G^{\prime}, M^{\prime}\right)$ may be obtained from $D(G, M)$ by cyclic twists and supertranspositions, and so $G^{\prime}$ may be obtained from $G$ by bi-twists and bi-transpositions.

\section{References}

[1] Richard A. Brualdi, Linear Preservers and Diagonal Hypergraphs. Linear Alg. Applics., 373 (2003), 51-65. 
[2] Richard A. Brualdi and Jeffrey A. Ross, Matrices with isomorphic diagonal hypergraphs. Discrete Math., 33 (1981), 123-138.

[3] Richard A. Brualdi and Herbert J. Ryser, Combinatorial Matrix Theory. Cambridge University Press, 1991.

[4] L. Lovász and M.D. Plummer, Matching Theory. Annals of Discrete Math., 29, NorthHolland, 1986.

[5] Carsten Thomassen, Whitney's 2-Switching Theorem, Cycle Space, and Arc Mappings of Directed Graphs. J. Comb. Theory Ser. B 46(3) (1989) 257-291.

[6] K. Truemper, On Whitney's 2-isomorphism theorem for graphs. J. Graph Theory (1980) 43-49.

[7] W.T. Tutte, Connectivity in Graphs. University of Toronto Press, Chapter 11, 1996.

[8] H. Whitney, 2-isomorphic graphs. Amer. J. Math. 55 (1933), 245-254. 